\title{
Uncertainty impairs grating detection performance in the cat
}

\author{
WILLIAM MARTENS and RANDOLPH BLAKE \\ Cresap Neuroscience Laboratory, Northwestern University, Evanston, Illinois 60201
}

\begin{abstract}
Using behavioral techniques, contrast thresholds for detection of grating patterns by the cat were measured under conditions of certainty and uncertainty. For certainty trials, the same combination of spatial and temporal frequencies was presented throughout a test session, whereas with uncertainty, the cat was unable to anticipate which of two spatial (or temporal) frequencies would be presented on a given trial. For most spatiotemporal combinations, uncertainty served to elevate contrast thresholds by almost . 3 log units, a finding that parallels the outcome found in human vision. The uncertainty paradigm may provide a useful inferential tool for determining channel bandwidth in cat vision.
\end{abstract}

On a variety of psychophysical tasks, detection performance is impaired when the subject is uncertain about some aspect of the stimulus event to be detected. For instance, in audition, the threshold for detection of pure sinusoids may be elevated twofold when the subject must listen for more than one tone (Green, 1964). In vision, threshold elevations of comparable magnitude have been found when observers are uncertain about the spatial location (Cohn \& Lasley, 1974) of a light increment, about the direction of motion of a moving target (Sekuler \& Ball, 1977), or about the spatial frequency of a grating pattern (Graham, Robson, \& Nachmias, 1978; Sekuler \& Tynan, 1978). While there are several possible interpretations of this so-called uncertainty effect, the most popular kind of model attributes the debilitating effects of uncertainty on detection performance to the existence of multiple detection channels which, under conditions of stimulus uncertainty, require some compromise monitoring strategy on the part of a subject. Building on this logic, uncertainty manipulations have been employed as an inferential tool for gauging the bandwidth of putative channels underlying detection; the logic of this strategy and its variations has been described in detail by Ball and Sekuler (Note 1).

In recent years, we have been studying spatial contrast vision in cats using psychophysical procedures (e.g., see Blake, 1978), the aim being to examine the extent to which the cat's visual performance can be related to receptive-field properties of visual neurons in the cat. In attempting to develop psychophysical strategies for measuring the spatial filtering charac-

This research was supported by grants from N.I.H. (EY01596) and N.S.F. (BNS7817948). R.B. is supported by a Career Development Award from N.I.H. (EY00106). We are grateful to Robert Sekuler for helpful discussion. Requests for reprints should be sent to R. Blake. teristics of detecting mechanisms in the cat, we decided to evaluate the effects of stimulus uncertainty on grating detection. The purpose of this note is to describe some of our preliminary findings along these lines.

\section{METHODS}

\section{Subjects}

Two adult female cats, designated as $B$ and $D$, served as subjects. Each had been trained and extensively tested in previous experiments on visual contrast sensitivity. During the course of behavioral testing, these cats were maintained on a 23-h fooddeprivation schedule, obtaining food only during daily experimental sessions.

\section{Apparatus and Visual Display}

The layout of the test apparatus and the particulars of the visual display have been described in detail elsewhere (Blake \& Camisa, 1977). In brief, during experimental sessions, the cat was housed in a restraining box which contained an aperture situated at head level at one end; this aperture was just large enough to allow the cat access to a lick tube located outside the box. This feeding device was situated in a way which forced the cat to maintain its head in a fixed position; a circular oscilloscope screen, $10 \mathrm{deg}$ in diameter, was located $50 \mathrm{~cm}$ directly in front of the cat's eyes. The space-average luminance of the raster generated on this screen was $60 \mathrm{~cd} / \mathrm{m}^{2}$; this raster could be modulated with sine-wave signals so as to produce either a stationary grating or one which flickered in counterphase. Gratings could be presented and withdrawn using a shaped rise/fall gate which varied the onset and offset of the modulating signal gradually over $250 \mathrm{msec}$ according to a Gaussian function; this mode of presentation served to eliminate abrupt transients. An infrared television camera, mounted immediately above the cat's head, provided a clear view of its eyes on a video monitor, and test trials were initiated only when the cat was looking at the display.

\section{Procedure}

In order to measure contrast thresholds, the cat was conditioned to suppress licking in the presence of a grating pattern which preceded a brief $(.25-\mathrm{sec})$ electric shock delivered to the cat's paws through the grid floor of the restraining box. This suppression behavior was quantified by comparing the number of licks made during a 10-sec presentation of the grating to the number of licks during a $10-\mathrm{sec}$ period immediately preceding the 
appearance of the grating. A $50 \%$ or greater reduction in lick rate during grating presentations was arbitrarily defined as detection.

Contrast thresholds were estimated using a staircase method (Cornsweet, 1962) whereby the contrast value presented on any given trial depended upon the cat's suppression performance on the previous trial. In our experiments, contrast was decreased following trials on which the cat detected the grating and was increased following trials on which the cat failed to detect the grating. At the beginning of each daily session, contrast was reduced in 6-dB steps $(.30 \log$ units) until the cat first failed to detect the grating, thereby leading to a reversal in the staircase. At this point, the staircase continued using a step size of $3 \mathrm{~dB}$. This smaller step size was not employed until the third reversal of the staircase, at which point grating contrast was varied in $1-\mathrm{dB}$ steps for the remainder of the $1-\mathrm{h}$ test session. Threshold was defined as the average contrast value over the final eight trials following the point at which the staircase reached an asymptotic level.

Contrast thresholds for vertical grating were measured at two spatial frequencies, .35 and 1.4 cycles/deg, with the grating either stationary or flickering in counterphase at $4 \mathrm{~Hz}$. These four thresholds were measured under two types of conditions, certainty and uncertainty. For the measurement of certainty thresholds, the same spatiotemporal combination was used on all trials in that session. For the spatial frequency uncertainty condition, the two spatial frequencies were randomly intermixed during a session by switching between the two staircase tracks unpredictably. Thus, the cat was unable to anticipate from trial to trial which spatial frequency would appear next. On these spatial frequency uncertainty trials, for a given session, the grating always appeared stationary $(0 \mathrm{~Hz})$ or always flickered in counterphase at $4 \mathrm{~Hz}$. For the temporal uncertainty condition, it was the temporal frequency $(0 \mathrm{vs} .4 \mathrm{~Hz}$ ) which was varied from trial to trial within a test session, which spatial frequency constant at either .35 or 1.4 cycles $/ \mathrm{deg}$.

\section{RESULTS}

Contrast sensitivity (reciprocal of contrast threshold) for the various spatiotemporal combinations under certainty and uncertainty conditions is shown for one cat in Figure 1; results from the other animal were qualitatively similar to these. For three of the four stimulus conditions, undertainty produced a marked reduction in sensitivity, on the order of $.20-.30 \mathrm{log}$ units. These differences in threshold between certainty and uncertainty far exceed the level of statistical significance, as the standard deviation of the mean contrast value at the asymptotic point in the staircase is only about .05 log units. Moreover, in replications of these kinds of threshold measurements, we routinely arrive at estimates that are within $1 \mathrm{~dB}$ of the original values. So we are confident that the uncertainty effect evidenced in these data is genuine.

It may be of significance that for both cats it was temporal uncertainty at .35 cycles/deg which failed to yield an uncertainty effect. To the extent that the effect of uncertainty stems from the existence of multiple detection channels, this result suggests that grating detection in the cat at this low spatial frequency may be mediated by a single mechanism with broad tuning for temporal fre-
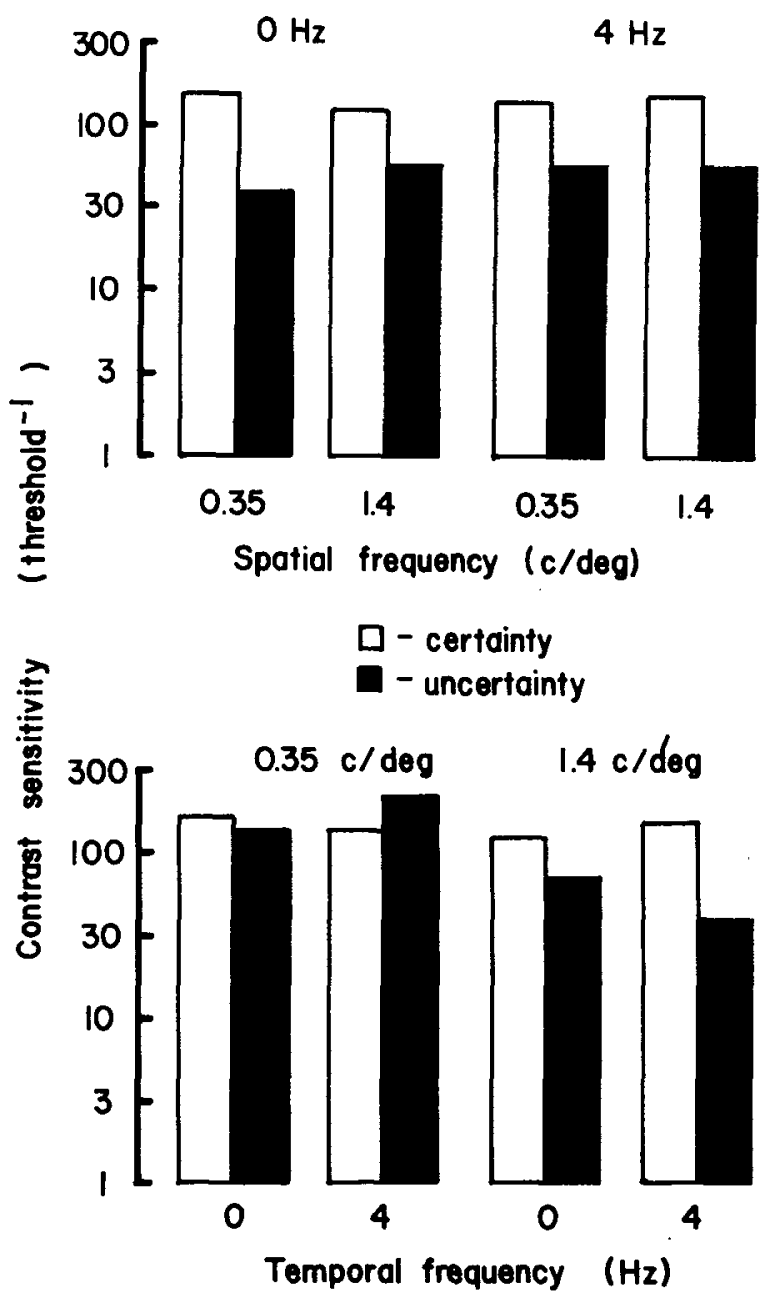

Figure 1. These histograms show the contrast sensitivity of cat $D$ for various combinations of spatial and temporal frequency. Thresholds were measured under conditions of certainty (wherein the same grating was presented on every trial of a session) and uncertainty (wherein two gratings, differing either in temporal frequency or spatial frequency, were randomly intermixed over trials). In this figure, the same data for the certainty conditions are represented twice, once in the upper panel and once in the lower panel, for purposes of graphic comparison.

quency. One might be tempted to look for physiological parallels within the context of the sustainedtransient dichotomy which currently is receiving much attention (e.g., see review by Rodieck, 1979). At this point, however, we are inclined simply to underscore the point that the influence of spatial uncertainty is not identical to the effects of temporal uncertainty.

\section{CONCLUSIONS}

The detection performance of the cat, just like that of man, suffers when there exists an element of uncertainty about the nature of the stimulus event 
to be detected. Of course, this finding does not necessarily shed more light on the specific basis of this uncertainty effect, but it does raise the possibility of a common underlying mechanism. There are those who have championed the viewpoint that uncertainty manifests itself via nonperceptual, response biases (e.g., Haber, 1966). To apply those arguments in the case of the cat would require attributing to the animal cognitive processes which ordinarily we reserve for animals higher on the phylogenetic scale.

This demonstration of an uncertainty effect in cat vision now sets the stage for using the uncertainty paradigm to estimate the bandwidths of the putative channels involved in detection, in a manner analogous to that described for human vision (Ball \& Sekuler, Note 1). Certainly, the magnitude of the effects of uncertainty on detection performance in the cat is sufficiently robust to provide a sensitive metric for these purposes.

\section{REFERENCE NOTE}

1. Ball, K., \& Sekuler, R. Models of stimulus uncertainty in motion perception. Manuscript in preparation.

\section{REFERENCES}

BlAKe, R. Spatial vision in the cat. In S. J. Cool \& E. L. Smith (Eds.), Frontiers in visual science. New York: Springer-Verlag, 1978.

Blake, R., \& Camisa, J. Temporal aspects of spatial vision in the cat. Experimental Brain Research, 1977, 28, 325-333.

Cohn, T. E., \& Lasley, D. J. Detectability of a luminance increment: Effect of spatial uncertainty. Journal of the Optical Society of America, 1974, 64, 1715-1719.

Cornsweet, T. N. The staircase method in psychophysics. American Journal of Psychology, 1962, 75, 485-491.

Graham, N., Robson, J. G., \& Nachmias, J. Grating summation in fovea and periphery. Vision Research, 1978, 18, 815-826.

GreEN, D. M. Detection of auditory sinusoids of uncertain frequency. In J. A. Swets (Ed.), Signal detection and recognition by human observers. New York: Wiley, 1964.

Haber, R. N. Nature of the effect of set on perception. Psychological Review, 1966, 73, 335-353.

RoDieck, R. W. Visual pathways. Annual Review of Neuroscience, 1979, 2, 193-225.

SEKULER, R., \& BALL, K. Mental set alters visibility of moving targets. Science, 1977, 198, 60-62.

Sekuler, R., \& Trnan, P. Rapid measurement of contrastsensitivity functions. American Journal of Optometry and Physiological Optics, 1978, 54, 573-575.

(Received for publication September 3, 1979; revision accepted September 13, 1979.) 